\title{
The effect of curvature on quasi-modes in coronal loops ${ }^{\star}$
}

\author{
T. Van Doorsselaere, A. Debosscher, J. Andries, and S. Poedts
}

\author{
Centre for Plasma Astrophysics, KU Leuven, Belgium \\ e-mail: tomvd@wis.kuleuven.ac.be
}

Received 6 May 2004 / Accepted 3 June 2004

\begin{abstract}
This paper studies quasi-mode oscillations in models of coronal loops that include longitudinal curvature. Using a toroidal coordinate system to incorporate curvature in a basic coronal loop model, the linearized ideal MHD equations are solved for the plasma- $\beta=0$. As a result of the curvature, quasi-modes with different poloidal wave numbers are coupled resulting in modifications of the frequencies. However, for small curvature, only the coupling of quasi-modes with a neighbouring poloidal wave number remains in first order. In addition, the quasi-mode frequencies are unchanged up to first order in the curvature. The imaginary part of the frequency, however, does change in first order, and quasi-modes are slightly more damped in realistically curved coronal loop configurations.
\end{abstract}

Key words. magnetohydrodynamics (MHD) - plasmas - Sun: corona - Sun: oscillations

\section{Introduction}

Coronal loop oscillations were first observed with TRACE by Schrijver et al. (1999); Aschwanden et al. (1999) and Nakariakov et al. (1999). Recently, Schrijver et al. (2002); Aschwanden et al. (2002) published the observational properties of 11 such events.

The nature of these lateral oscillations is believed to be a kink $(m=1)$ quasi-mode oscillation. Analytical calculations in cylindrical configurations of the frequency and damping times of such quasi-modes were published by Hollweg \& Yang (1988), Goossens et al. (1992) and Goossens et al. (1995). In these analytical derivations, it is assumed that the radial inhomogeneity is limited to a thin boundary layer. Numerical calculations concerning thick boundaries were done by Hollweg (1990) and Van Doorsselaere et al. (2004). The latter authors showed that the use of the analytical formula for the damping times (Ruderman \& Roberts 2002) may be extended to the "thick" boundary limit.

The observed oscillations also provide an excellent tool for coronal seismology. Nakariakov \& Ofman (2001) used them to obtain an estimate for the value of the magnetic field in coronal loops and Goossens et al. (2002a) and Aschwanden et al. (2003) exploited these oscillations to calculate the density ratio (internal/external) for a set of loops and compared it with the observed values. The agreement between the observed and calculated values was within a factor 2 , which is excellent, given the observational constraints.

Most authors use a straight cylindrically symmetric density enhancement in a straight homogeneous magnetic field as a model for a coronal loop to determine the quasi-mode frequencies. An overview of recent results can be found in Goossens et al. (2002b). Effects due to longitudinal curvature are almost always neglected, because they are believed to be small.

In a different context, however, Kieras \& Tataronis (1982) calculated that "toroidal" curvature has drastic effects on continuum modes in tokamaks. These effects include avoided crossing and the appearance of new modes due to the toroidicity (TAE). These authors showed that, for small aspect ratios (small radius/ large radius), these Alfvén continuum modes only couple to eigenmodes with neighbouring poloidal mode numbers. However, it is unclear whether this effect is similar for ideal quasi-modes in coronal loops.

In this paper, we use appropriate toroidal coordinates which have toroidal curvature built in to model the quasi-modes in semi-toroidal coronal loops. We then solve the linearized ideal MHD equations using these toroidal coordinates.

In Sect. 2 we introduce the coordinate system and the equations, and we specify the equilibrium that we use to model a coronal loop. Section 3 is dedicated to solving the governing differential equation for the linear perturbations. In Sect. 4, we investigate the effects of small curvature. In Sect. 5 a smoothly varying density profile is added, resulting in resonant damping of the quasi-mode. In Sect. 6, we investigate the case of, as far as the curvature is concerned, realistic coronal loops. A conclusion can be found in Sect. 7.

\footnotetext{
* Appendix A is only available in electronic form at http://www.edpsciences.org
} 


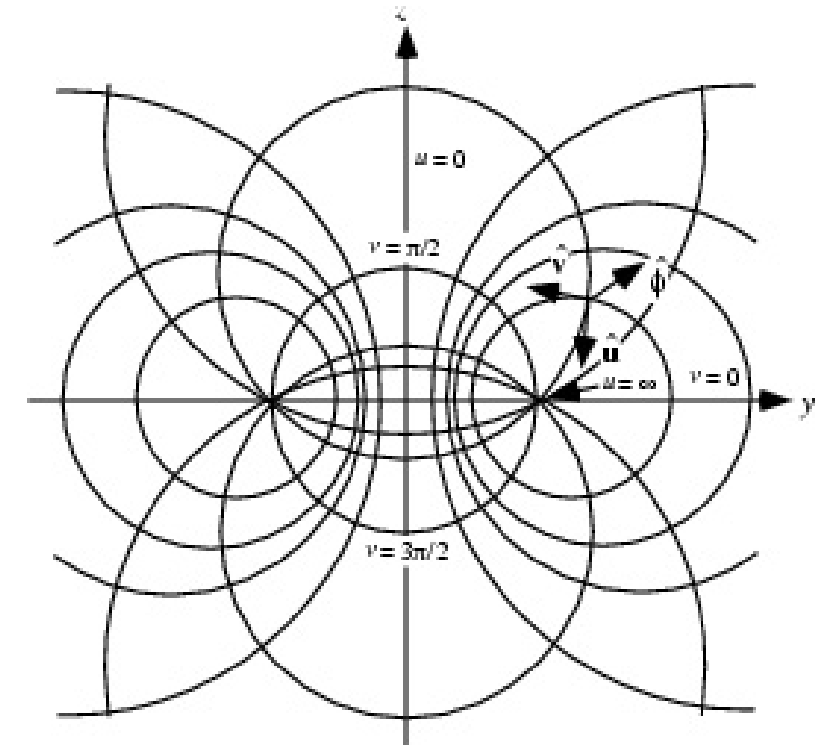

Fig. 1. Toroidal coordinate system.

\section{Coordinate system, equations and equilibrium}

In order to describe the effect of longitudinal curvature in coronal loops, a toroidal coordinate system is used. This coordinate system is defined by the following transformation formulae:

$x=\frac{a \sinh u \cos \phi}{\cosh u-\cos v}, \quad y=\frac{a \sinh u \sin \phi}{\cosh u-\cos v}, \quad z=\frac{a \sin v}{\cosh u-\cos v}$.

Here $u, v$ and $\phi$ are respectively the radial coordinate, the poloidal coordinate (small circle), and the toroidal coordinate. $a$ is a scaling parameter and determines the large radius of the torus. A sketch of the resulting coordinate lines can be found in Fig. 1.

In this toroidal coordinate system, constant $u$-surfaces are nested tori. We use $u=u_{0}$ as the boundary of the model of the coronal loop. All quantities inside the coronal loop $\left(u>u_{0}\right)$ are denoted with subscript $\mathrm{i}$ and all quantities in the outer region $\left(u<u_{0}\right)$ by e. In our equilibrium model, we use a continuous magnetic field directed along the toroidal axis of the loop, whereas the density has a discontinuous jump at $u=u_{0}$. We thus model a coronal loop as a toroidal density enhancement embedded in a "straight" magnetic field (i.e. a field with constant curvature).

We shall solve the linearized ideal MHD equations in the internal and external region to calculate the frequency and damping rate of quasi-modes. The use of the linearized equations may be justified by the fact that the typical velocity perturbations are much smaller than the estimated Alfvén speed (Aschwanden et al. 2002).

In our model, we neglect gravity and gas pressure. The latter may be justified by the fact that the plasma- $\beta$ is very small in the corona. As a result of these assumptions, we exclude the slow magnetosonic waves from the analysis. We also assume that there is no equilibrium velocity. Consequently, the governing equations for the equilibrium reduce to

$\frac{1}{\mu}(\nabla \times B) \times \boldsymbol{B}=0$
$\nabla \cdot \boldsymbol{B}=0$.

Here $\boldsymbol{B}$ denotes the magnetic field and $\mu$ is the magnetic permeability.

We can satisfy the solenoidal constraint (Eq. (2)) by considering a $2 \mathrm{D}$ equilibrium with $\boldsymbol{B}=B(u, v) \boldsymbol{e}_{\phi}$, where $\boldsymbol{e}_{\phi}$ is the unit vector in the toroidal (ignorable) direction. With this form of the magnetic field, the force-free condition is equivalent to the current-free condition.

We neglect poloidal magnetic fields, and thus field-aligned currents, because Goedbloed \& Halberstadt (1994) showed that the Alfvén continuum is not influenced by this magnetic field component in coronal loop models with line-tying boundary conditions.

The force-free equation (Eq. (1)) has a unique solution:

$B(u, v)=B_{\mathrm{a}} \frac{(\cosh u-\cos v)}{\sinh u}$.

Here is $B_{\mathrm{a}}$ the value of the magnetic field on the axis of the torus. Rewritten in Cartesian coordinates, we have $B \sim \frac{1}{r}=\frac{1}{\sqrt{x^{2}+y^{2}}}$. This magnetic field has a singularity on the z-axis, i.e. the axis perpendicular to the plane of the coronal loop axis. This equilibrium 


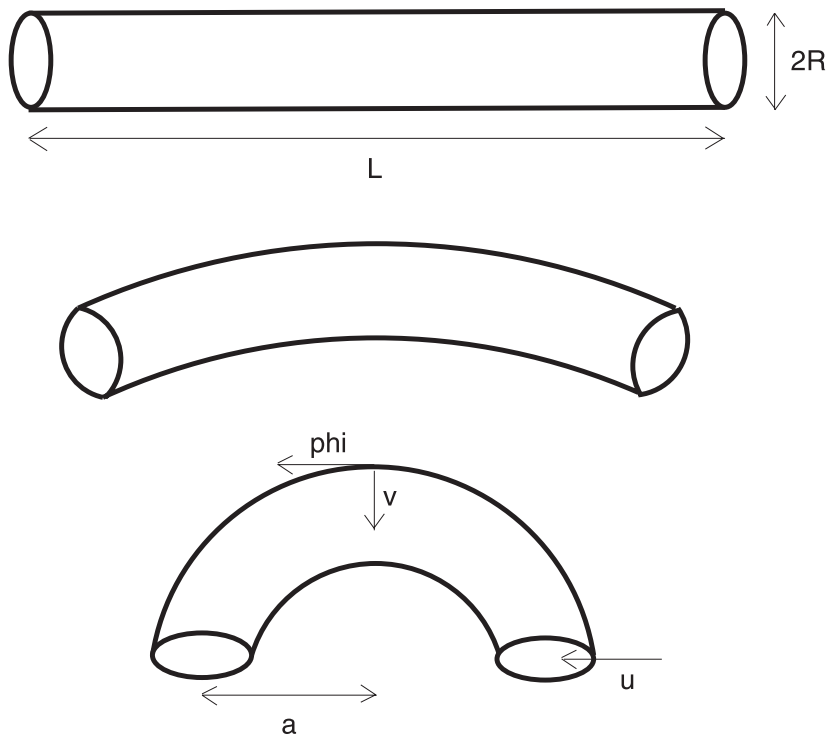

Fig. 2. Our model for different $k$-values. By adjusting $k$, we determine how the coronal loop intersects with the chromosphere. $L$ is the length of the loop, $R$ the small radius, $a$ the large radius.

magnetic field corresponds to a magnetic field around a current carrying wire. However, the region around the $z$-axis is of no importance, because we are only interested in the region in the vicinity of the coronal loop. Furthermore, upon solving the linearized MHD equations in the external region, we apply regularity conditions so that one of the two solutions can be rejected (see Sect. 3.1). Posing the regularity conditions could be a problem because it interferes with the equilibrium singularity at the $z$-axis. However, because $u=0$ corresponds to both the $z$-axis as well as $r=\sqrt{x^{2}+y^{2}} \rightarrow \infty$, the regularity conditions can be applied at the latter location instead of at the z-axis. We can thus avoid the equilibrium singularity at the $z$-axis and the singular behaviour will pose no problem when solving the linearized MHD equations.

We remind that the equilibrium density $\rho$ can still be chosen arbitrarily since we neglected gravity.

We now have an equilibrium model which is similar to the one used by Ruderman \& Roberts (2002) and Van Doorsselaere et al. (2004). This will facilitate the comparison between the cylindrical and the curved configurations.

Since the equilibrium magnetic field (Eq. (3)) is current-free, the governing equations for the linear perturbations are

$\rho \frac{\partial \boldsymbol{V}}{\partial t}=\frac{1}{\mu}(\boldsymbol{\nabla} \times \boldsymbol{b}) \times \boldsymbol{B}$

$\frac{\partial \boldsymbol{b}}{\partial t}=\boldsymbol{\nabla} \times(\boldsymbol{V} \times \boldsymbol{B})$,

where $\boldsymbol{b}=\left(b_{u}, b_{v}, b_{\phi}\right)$ and $\boldsymbol{V}=\left(V_{u}, V_{v}, V_{\phi}\right)$ are the perturbations of the magnetic field and the velocity respectively. The other linearized MHD equations are completely decoupled from the given ones and will not be used in what follows.

Expanding these equations in toroidal coordinates, we have

$$
\begin{aligned}
& \frac{\partial^{2} \boldsymbol{V}}{\partial t^{2}}+\omega_{\mathrm{A}}^{2} \boldsymbol{V}=V_{\mathrm{A}}^{2} \boldsymbol{\nabla}_{\perp}\left(\frac{1}{B} \frac{\partial b_{\phi}}{\partial t}\right), \\
& \frac{\partial b_{\phi}}{\partial t}=-\frac{B}{a} \sinh u(\cosh u-\cos v)\left\{\frac{\partial}{\partial u}\left(\frac{V_{u}}{\sinh u}\right)+\frac{\partial}{\partial v}\left(\frac{V_{v}}{\sinh u}\right)\right\} .
\end{aligned}
$$

Because the equilibrium quantities are independent of $\phi$, we have Fourier analyzed the perturbations in this direction and have put all perturbed quantities proportional to $\exp (i k \phi)$, where $k$ is the wave number in the $\phi$-direction. The fundamental mode can be modelled by taking two consecutive nodes as the footpoints of the coronal loop. By changing the wavenumber $k$, we can adjust the intersection of the coronal loop with the chromosphere (see Fig. 2). Perfect semi-circular coronal loops correspond to $k=1$ and cylindrical coronal loop models are obtained for $k \rightarrow \infty$.

Additionally, we define the Alfvén speed $V_{\mathrm{A}}^{2}=B^{2} / \mu \rho$ and the Alfvén frequency $\omega_{\mathrm{A}}^{2}=\frac{V_{A}^{2} k^{2}(\cosh u-\cos v)^{2}}{a^{2} \sinh ^{2} u}$. We also have $\boldsymbol{\nabla}_{\perp}=\boldsymbol{\nabla}-\boldsymbol{e}_{\phi}\left(\frac{\cosh u-\cos v}{a \sinh u} \frac{\partial}{\partial \phi}\right)$, which is the gradient in the cross-section of the coronal loop perpendicular to the axis.

Our coronal loop model is summarized in Fig. 2. We model a coronal loop with given length $L$ and small radius $R$ with an appropriate $a$ (large radius), $k$ and $u_{0}$ (boundary of the loop). Everywhere in space, we use the equilibrium magnetic field given by Eq. (3), whereas the density is piecewise constant, having a jump at $u=u_{0}$. 
Since we consider a static equilibrium, we can Fourier analyze the time dependency and put all perturbed quantities proportional to $\exp (-\mathrm{i} \omega t)$. Consequently, we are looking for normal eigenmodes. After considerable calculations, we can obtain a single equation for the dependent variable $b_{\phi} / B$ from Eqs. (4) and (5):

$$
\begin{aligned}
-\frac{b_{\phi}}{B}= & \frac{\omega_{\mathrm{A}}^{2} \sinh ^{2} u}{k^{2}\left(\omega^{2}-\omega_{\mathrm{A}}^{2}\right)}\left\{\frac{\partial^{2}}{\partial u^{2}}\left(\frac{b_{\phi}}{B}\right)+\frac{\partial^{2}}{\partial v^{2}}\left(\frac{b_{\phi}}{B}\right)\right. \\
& +\left(\frac{\cosh u \cos v-1}{\sinh u(\cosh u-\cos v)}\left(\frac{3 \omega^{2}+\omega_{\mathrm{A}}^{2}}{\omega^{2}-\omega_{\mathrm{A}}^{2}}\right)-\left(\frac{\omega^{2}}{\omega^{2}-\omega_{\mathrm{A}}^{2}}\right) \frac{\partial \ln \rho}{\partial u}\right) \frac{\partial}{\partial u}\left(\frac{b_{\phi}}{B}\right) \\
& \left.+\left(\frac{\sin v}{\cosh u-\cos v}\left(\frac{3 \omega^{2}+\omega_{\mathrm{A}}^{2}}{\omega^{2}-\omega_{\mathrm{A}}^{2}}\right)-\left(\frac{\omega^{2}}{\omega^{2}-\omega_{\mathrm{A}}^{2}}\right) \frac{\partial \ln \rho}{\partial v}\right) \frac{\partial}{\partial v}\left(\frac{b_{\phi}}{B}\right)\right\} .
\end{aligned}
$$

As an equilibrium density profile, we choose $\rho(u, v)=\rho_{\mathrm{a}}\left(\frac{\cosh u-\cos v}{\sinh u}\right)^{4}$ such that $\omega_{\mathrm{A}}$ becomes independent of both $u$ and $v$. Like the equilibrium magnetic field, this density distribution has a strong singularity $\left(\sim \frac{1}{r^{4}}\right)$ at the $z$-axis, but similar to the equilibrium magnetic field this singularity can be circumvented when applying the boundary conditions.

With this choice of density distribution, we can reduce Eq. (6) to

$\nabla_{\perp}^{2}\left(\frac{b_{\phi}}{B}\right)=-\left(\frac{k^{2}\left(\omega^{2}-\omega_{\mathrm{A}}^{2}\right)(\cosh u-\cos v)^{2}}{a^{2} \omega_{\mathrm{A}}^{2} \sinh ^{2} u}\right) \frac{b_{\phi}}{B}$,

or

$\nabla^{2}\left(\frac{b_{\phi}}{B}\right)=-\left(\frac{k^{2} \omega^{2}(\cosh u-\cos v)^{2}}{\omega_{\mathrm{A}}^{2} a^{2} \sinh ^{2} u}\right) \frac{b_{\phi}}{B}$

These are just classical wave equations with space dependent wave speed. Solving them will yield the perturbed magnetic field in regions with constant Alfvén frequency.

\section{Solutions in regions with constant Alfvén frequency}

We want to solve Eq. (7) in order to find the eigenfunctions of the quasi-modes.

To make the equation separable, we substitute

$$
\frac{b_{\phi}}{B}=\sqrt{\frac{\cosh u-\cos v}{\sinh u}} l(u, v),
$$

and find, after division by $\frac{1}{a^{2}} \sqrt{\frac{\cosh u-\cos v}{\sinh u}}(\cosh u-\cos v)^{2}$, the differential equation for $l$ as:

$$
\frac{\partial^{2} l}{\partial u^{2}}+\frac{\partial^{2} l}{\partial v^{2}}+\left(k^{2} \frac{\omega^{2}}{\omega_{\mathrm{A}}^{2}}-k^{2}+\frac{1}{4}\right) \frac{1}{\sinh ^{2} u} l=0,
$$

which is clearly separable. We take $l(u, v)=\mathbb{U}(u) \mathbb{V}(v)$ to find

$$
\frac{1}{\mathbb{U}} \frac{\partial^{2} \mathbb{U}}{\partial u^{2}}+\left(k^{2} \frac{\omega^{2}}{\omega_{\mathrm{A}}^{2}}-k^{2}+\frac{1}{4}\right) \frac{1}{\sinh ^{2} u}=-\frac{1}{\mathbb{V}} \frac{\partial^{2} \mathbb{V}}{\partial v^{2}}=\beta^{2},
$$

with $\beta$ a separation constant. It follows that $\mathbb{V}(v)$ is a linear combination of $\exp \left( \pm \mathrm{i} \beta\left(v-v_{0}\right)\right)$, with $v_{0}$ an arbitrary phase. Because $v$ is a periodical coordinate, $\beta$ must be an integer. $\beta$ is the poloidal wave number, similar to $m$ in the cylindrical case. Since $v_{0}$ is arbitrary, there is still no preferential oscillation direction. Depending on the direction of the triggering disturbance, an appropriate phase may be chosen. In contrast to what the observations suggest (Schrijver et al. 2002), not only oscillations perpendicular to the plane of the loop are possible in our equilibrium, no preferential direction is introduced in our curved configuration.

The radial solution $\mathbb{U}(u)$ can be expressed in terms of hypergeometric functions.

We now define a jump for the density profile. In the internal region $u>u_{0}$ we have $\rho_{\mathrm{a}}=\rho_{\mathrm{a}, \mathrm{i}}$. In external region $u<u_{0}$, analogously $\rho_{\mathrm{a}}=\rho_{\mathrm{a}, \mathrm{e}}$. The case of overdense coronal loops (as observed by Aschwanden et al. 2003) corresponds to $\rho_{\mathrm{a}, \mathrm{i}}>\rho_{\mathrm{a}, \mathrm{e}}$.

We now have two regions with constant Alfvén frequency where a solution can be found. Matching the internal and external solutions leads to a dispersion relation, which determines the eigenfrequencies and the quasi-modes. 


\subsection{External region}

After defining $\alpha^{2}=\omega^{2} / \omega_{\mathrm{A}}^{2}$, two valid linearly independent solutions in the external region are

$\mathbb{U}_{1}(u, \beta, k)=(\cosh u)^{-\beta}(\operatorname{coth} u)^{-\frac{1}{2}-k \sqrt{1-\alpha_{\mathrm{e}}^{2}}}{ }_{2} F_{1}\left(\frac{1}{4}+\frac{k}{2} \sqrt{1-\alpha_{\mathrm{e}}^{2}}+\frac{\beta}{2}, \frac{3}{4}+\frac{k}{2} \sqrt{1-\alpha_{\mathrm{e}}^{2}}+\frac{\beta}{2} ; 1+k \sqrt{1-\alpha_{\mathrm{e}}^{2}} ; \tanh ^{2} u\right)$,

$\mathbb{U}_{2}(u, \beta, k)=\mathbb{U}_{1}(u,-\beta,-k)$,

where ${ }_{2} F_{1}(a, b ; c ; z)=\sum_{n=0}^{\infty} \frac{(a)_{n}(b)_{n}}{(c)_{n}} z^{n}$ is the hypergeometric function and $(a)_{n}=\frac{\Gamma(a+n)}{\Gamma(a)}$.

We can now apply regularity conditions at infinity $\left(r=\sqrt{x^{2}+y^{2}} \rightarrow \infty\right)$, which corresponds to $u=0$. Of these two solutions, we have to reject $\mathbb{U}_{2}(u)$, because for at least some values of $\alpha_{\mathrm{e}}$ it has a singularity at $u=0$. In what follows, we will refer to $\mathbb{U}_{1}(u)$ as $F_{\mathrm{e}, \beta}(u)$.

\subsection{Internal region}

Finding a solution in the internal region is somewhat more difficult. Depending on the sign of $\beta$, we have to use a different solution. However, in each case, we have to reject one solution because of regularity conditions on the axis of the torus $(u \rightarrow \infty)$. In order to keep the poloidal speed $V_{v}$ finite, $b_{\phi} / B$ must tend to 0 as $u \rightarrow \infty$. The resulting solution in the internal region is:

$F_{\mathrm{i}, \beta}(u)=(\cosh u)^{-|\beta|}(\operatorname{coth} u)^{-\frac{1}{2}+k \sqrt{1-\alpha_{\mathrm{i}}^{2}}}{ }_{2} F_{1}\left(\frac{1}{4}-\frac{k}{2} \sqrt{1-\alpha_{\mathrm{i}}^{2}}+\frac{|\beta|}{2}, \frac{3}{4}-\frac{k}{2} \sqrt{1-\alpha_{\mathrm{i}}^{2}}+\frac{|\beta|}{2} ; 1+|\beta| ; \frac{1}{\cosh ^{2} u}\right)$.

\subsection{Matching the solutions}

A general solution of Eq. (7) is (apart from a separation constant, see Eq. (8)) a superposition of the basic solutions and is given as $\sqrt{\frac{\cosh u-\cos v}{\sinh u}} \sum_{\beta=-\infty}^{+\infty} C_{\mathrm{i} / \mathrm{e}, \beta} F_{\mathrm{i} / \mathrm{e}, \beta}(u) \exp \left(i \beta\left(v-v_{0}\right)\right)$.

We demand that $b_{\phi} / B=\mu P^{\prime} / B^{2}$ ( $P^{\prime}$ is the perturbed pressure, cf. cylindrical case) and $V_{u}$ are continuous across the discontinuity interface at $u=u_{0}$. This yields a system of equations for the amplitudes of the different solutions $C_{\mathrm{i} / \mathrm{e}, \beta}$ at $u=u_{0}$ :

$\sum_{\beta=-\infty}^{+\infty} C_{\mathrm{e}, \beta} F_{\mathrm{e}, \beta}\left(u_{0}\right) \exp \left(\mathrm{i} \beta\left(v-v_{0}\right)\right)=\sum_{\beta=-\infty}^{+\infty} C_{\mathrm{i}, \beta} F_{\mathrm{i}, \beta}\left(u_{0}\right) \exp \left(\mathrm{i} \beta\left(v-v_{0}\right)\right)$,

$\sum_{\beta=-\infty}^{+\infty} \frac{\exp \left(\mathrm{i} \beta\left(v-v_{0}\right)\right)}{\alpha_{\mathrm{e}}^{2}-1} C_{\mathrm{e}, \beta}\left(\frac{\mathrm{d} F_{\mathrm{e}, \beta}}{\mathrm{d} u}\left(u_{0}\right)+F_{\mathrm{e}, \beta}\left(u_{0}\right) \frac{1}{2} \frac{\cos v \cosh u_{0}-1}{\sinh u_{0}\left(\cosh u_{0}-\cos v\right)}\right)$

$$
=\sum_{\beta=-\infty}^{+\infty} \frac{\exp \left(\mathrm{i} \beta\left(v-v_{0}\right)\right)}{\alpha_{\mathrm{i}}^{2}-1} C_{\mathrm{i}, \beta}\left(\frac{\mathrm{d} F_{\mathrm{i}, \beta}}{\mathrm{d} u}\left(u_{0}\right)+F_{\mathrm{i}, \beta}\left(u_{0}\right) \frac{1}{2} \frac{\cos v \cosh u_{0}-1}{\sinh u_{0}\left(\cosh u_{0}-\cos v\right)}\right) .
$$

We multiply these equations with $\exp \left(-\mathrm{i} \beta^{\prime} v\right)$ and integrate over $v$, i.e. we Fourier-analyze the equations with respect to $v$, yielding: $0=C_{\mathrm{e}, \beta^{\prime}} F_{\mathrm{e}, \beta^{\prime}}\left(u_{0}\right)-C_{\mathrm{i}, \beta^{\prime}} F_{\mathrm{i}, \beta^{\prime}}\left(u_{0}\right)$,

$0=\sum_{\delta=-\infty}^{+\infty} \frac{1}{\alpha_{\mathrm{e}}^{2}-1} C_{\mathrm{e}, \beta^{\prime}} \frac{\mathrm{d} F_{\mathrm{e}, \beta^{\prime}}}{\mathrm{d} u}\left(u_{0}\right)-\frac{1}{\alpha_{\mathrm{i}}^{2}-1} C_{\mathrm{i}, \beta^{\prime}} \frac{\mathrm{d} F_{\mathrm{i}, \beta^{\prime}}}{\mathrm{d} u}\left(u_{0}\right)-\frac{\operatorname{coth} u_{0}}{2}\left(\frac{C_{\mathrm{e}, \beta^{\prime}} F_{\mathrm{e}, \beta^{\prime}}\left(u_{0}\right)}{\alpha_{\mathrm{e}}^{2}-1}-\frac{C_{\mathrm{i}, \beta^{\prime}} F_{\mathrm{i}, \beta^{\prime}}\left(u_{0}\right)}{\alpha_{\mathrm{i}}^{2}-1}\right)$

$$
+\frac{g_{\delta}}{2} \exp \left(-\mathrm{i} \delta v_{0}\right)\left(\frac{C_{\mathrm{e}, \beta^{\prime}+\delta} F_{\mathrm{e}, \beta^{\prime}+\delta}\left(u_{0}\right)}{\alpha_{\mathrm{e}}^{2}-1}-\frac{C_{\mathrm{i}, \beta^{\prime}+\delta} F_{\mathrm{i}, \beta^{\prime}+\delta}\left(u_{0}\right)}{\alpha_{\mathrm{i}}^{2}-1}\right),
$$

where $\delta=\beta-\beta^{\prime}$ and $g_{\delta}=\frac{1}{2 \pi} \int_{0}^{2 \pi} \frac{\exp (\mathrm{i} \delta v) \sinh u_{0}}{\cosh u_{0}-\cos v} \mathrm{~d} v=\exp \left(-|\delta| u_{0}\right)$. This is an infinite system of coupled equations for the $C_{\mathrm{i} / \mathrm{e}, \beta^{\prime}}$. Therefore, modes are not characterized by a single poloidal wave number, but are a superposition of interacting waves with different poloidal wave numbers.

With the aid of Eq. (12), we can eliminate $C_{\mathrm{e}, \beta^{\prime}}$ from Eq. (13). Replacing $\beta^{\prime}$ with $\beta$ then yields

$$
\begin{aligned}
0= & C_{\mathrm{i}, \beta}\left(\left(\alpha_{\mathrm{i}}^{2}-1\right) \frac{\mathrm{d} \log F_{\mathrm{e}, \beta}}{\mathrm{d} u}\left(u_{0}\right)-\left(\alpha_{\mathrm{e}}^{2}-1\right) \frac{\mathrm{d} \log F_{\mathrm{i}, \beta}}{\mathrm{d} u}\left(u_{0}\right)+\frac{1-\operatorname{coth} u_{0}}{2}\left(\alpha_{\mathrm{i}}^{2}-\alpha_{\mathrm{e}}^{2}\right)\right) \\
& +\sum_{\delta \neq 0} C_{\mathrm{i}, \beta+\delta} \exp \left(-\mathrm{i} \delta v_{0}\right) \frac{\exp \left(-|\delta| u_{0}\right)}{2} \frac{F_{\mathrm{i}, \beta+\delta}\left(u_{0}\right)}{F_{\mathrm{i}, \beta}\left(u_{0}\right)}\left(\alpha_{\mathrm{i}}^{2}-\alpha_{\mathrm{e}}^{2}\right) .
\end{aligned}
$$


It is possible to solve this (infinite) system numerically for the $C_{\mathrm{i}, \beta}$ coefficients. However, analytical progress is still possible when we consider the limit of small curvature since in that case only a few terms survive in the system (14).

\section{First-order approximation}

We want to examine the limit of small curvature in order to compare our results with the cylindrical solutions. As a small parameter $\varepsilon$ we take $R / a$, where $R$ is the radius of the coronal loop. When $a$ goes to infinity, we have to change $u_{0}$ so that the radius $R$ remains constant. Additionally, we have to increase $k$ to keep the length of the coronal loop constant. The following relationships are obtained:

$\varepsilon=\frac{R}{a}=\frac{\pi R}{L k}=\frac{1}{\sinh u_{0}}$

We have (when only retaining the two dominant terms in the expansion with respect to $\varepsilon$ )

$\tanh u_{0} \approx 1-\frac{\varepsilon^{2}}{2}, \quad \operatorname{coth} u_{0} \approx 1+\frac{\varepsilon^{2}}{2}, \quad \exp \left(-u_{0}\right) \approx \frac{\varepsilon}{2}$

$C_{\mathrm{i} / \mathrm{e}, \beta}$ also depends on $\varepsilon$. We find this dependency by looking at the zeroth order of the solution in the radial direction. Expanding the radial solution in $\varepsilon$ shows us that the internal solution has a $C_{\mathrm{i} / \mathrm{e}, \beta} \varepsilon^{|\beta|}\left(F_{0}+\varepsilon F_{1}\right)$ dependency where the

$$
F_{0}={ }_{0} F_{1}\left(; 1+|\beta| ;\left(\frac{\pi R}{2 L}\right)^{2}\left(1-\alpha_{\mathrm{i}}^{2}\right)\right)=\frac{\Gamma(1+|\beta|) I_{|\beta|}\left(\frac{\pi R}{L} \sqrt{1-\alpha_{\mathrm{i}}^{2}}\right)}{\left(\frac{\pi R}{2 L} \sqrt{1-\alpha_{\mathrm{i}}^{2}}\right)^{|\beta|}} .
$$

Hence, in order to obtain a solution which is in zeroth-order equal to a Bessel function (cf. cylindrical case), we have to take $C_{\mathrm{i} / \mathrm{e}, \beta} \sim \varepsilon^{-|\beta|}$. We now define new constants $D_{\mathrm{i} / \mathrm{e}, \beta}=C_{\mathrm{ie}, \beta} \varepsilon^{|\beta|}$, which is of the zeroth order in $\varepsilon$.

Rewriting Eq. (14) in terms of $D_{\mathrm{i} / \mathrm{e}, \beta}$ gives

$$
\begin{aligned}
0= & D_{\mathrm{i}, \beta}\left(\left(\alpha_{\mathrm{i}}^{2}-1\right) \frac{\mathrm{d} \log F_{\mathrm{e}, \beta}}{\mathrm{d} u}\left(u_{0}\right)-\left(\alpha_{\mathrm{e}}^{2}-1\right) \frac{\mathrm{d} \log F_{\mathrm{i}, \beta}}{\mathrm{d} u}\left(u_{0}\right)+\frac{1-\operatorname{coth} u_{0}}{2}\left(\alpha_{\mathrm{i}}^{2}-\alpha_{\mathrm{e}}^{2}\right)\right) \\
& +\sum_{\delta \neq 0} D_{\mathrm{i}, \beta+\delta} \varepsilon^{|\beta|-|\beta+\delta|} \exp \left(-\mathrm{i} \delta v_{0}\right) \frac{\exp \left(-|\delta| u_{0}\right)}{2} \frac{F_{\mathrm{i}, \beta+\delta}\left(u_{0}\right)}{F_{\mathrm{i}, \beta}\left(u_{0}\right)}\left(\alpha_{\mathrm{i}}^{2}-\alpha_{\mathrm{e}}^{2}\right) .
\end{aligned}
$$

We now expand this equation in $\varepsilon$. First we expand $\frac{\mathrm{d} \log F_{\mathrm{i}, \beta}}{\mathrm{d} u}\left(u_{0}\right)$.

$$
\begin{aligned}
\frac{\mathrm{d} \log F_{\mathrm{i}, \beta}}{\mathrm{d} u}= & \frac{\mathrm{d}}{\mathrm{d} u}\left\{-|\beta| \log \cosh u-\left(\frac{1}{2}-k \sqrt{1-\alpha_{\mathrm{i}}^{2}}\right) \log \operatorname{coth} u\right. \\
& \left.+\log _{2} F_{1}\left(\frac{1}{4}-\frac{k}{2} \sqrt{1-\alpha_{\mathrm{i}}^{2}}+\frac{|\beta|}{2}, \frac{3}{4}-\frac{k}{2} \sqrt{1-\alpha_{\mathrm{i}}^{2}}+\frac{|\beta|}{2} ; 1+|\beta| ; \frac{1}{\cosh ^{2} u}\right)\right\} \\
= & -|\beta|-2 \frac{X_{0}}{1+|\beta|}\left(\frac{\pi R}{2 L}\right)^{2}\left(1-\alpha_{\mathrm{i}}^{2}\right) \\
& +\varepsilon\left(-\frac{\pi R}{L} \sqrt{1-\alpha_{\mathrm{i}}^{2}}-2 \frac{X_{0}}{1+|\beta|}\left[X_{1}\left(\frac{\pi R}{2 L}\right)^{2}\left(1-\alpha_{\mathrm{i}}^{2}\right)-(1+|\beta|) \frac{\pi R}{2 L} \sqrt{1-\alpha_{\mathrm{i}}^{2}}\right]\right),
\end{aligned}
$$

where

$$
\begin{aligned}
X_{0}= & \frac{\sum_{n=0}^{\infty} \frac{1}{n !(2+|\beta|)_{n}}\left(\frac{\pi R}{2 L}\right)^{2 n}\left(1-\alpha_{\mathrm{i}}^{2}\right)^{n}}{\sum_{n=0}^{\infty} \frac{1}{n !(1+|\beta|)_{n}}\left(\frac{\pi R}{2 L}\right)^{2 n}\left(1-\alpha_{\mathrm{i}}^{2}\right)^{n}}=\frac{{ }_{0} F_{1}\left(; 2+|\beta| ;\left(\frac{\pi R}{2 L}\right)^{2}\left(1-\alpha_{\mathrm{i}}^{2}\right)\right)}{{ }_{0} F_{1}\left(; 1+|\beta| ;\left(\frac{\pi R}{2 L}\right)^{2}\left(1-\alpha_{\mathrm{i}}^{2}\right)\right)}, \\
X_{1}= & \frac{1}{{ }_{0} F_{1}\left(; 1+|\beta| ;\left(\frac{\pi R}{2 L}\right)^{2}\left(1-\alpha_{\mathrm{i}}^{2}\right)\right)} \sum_{n=0}^{\infty} \frac{1}{n !(1+|\beta|)_{n}}\left(\frac{\pi R}{2 L}\right)^{2 n}\left(1-\alpha_{\mathrm{i}}^{2}\right)^{n} \frac{2 n L(|\beta|+n)}{R \pi \sqrt{1-\alpha_{\mathrm{i}}^{2}}} \\
& -\frac{1}{{ }_{0} F_{1}\left(; 2+|\beta| ;\left(\frac{\pi R}{2 L}\right)^{2}\left(1-\alpha_{\mathrm{i}}^{2}\right)\right)} \sum_{n=0}^{\infty} \frac{1}{n !(2+|\beta|)_{n}}\left(\frac{\pi R}{2 L}\right)^{2 n}\left(1-\alpha_{\mathrm{i}}^{2}\right)^{n} \frac{2 n L(|\beta|+n+2)}{R \pi \sqrt{1-\alpha_{\mathrm{i}}^{2}}} .
\end{aligned}
$$

It is possible to prove that the first order correction to $\frac{\mathrm{d} \log F_{\mathrm{i}, \beta}}{\mathrm{d} u}\left(u_{0}\right)$ is zero. 
Similarly, we can prove that $\frac{\mathrm{d} \log F_{\mathrm{e}, \beta}}{\mathrm{d} u}\left(u_{0}\right)$ is zero. In this case, however, we have to distinguish between different cases, depending on the sign of $\beta$. For more details, see Appendix A.

We have not been able to prove analytically that the first order contribution to this term is also zero, but our numerical calculations yield, for the first order, values smaller than $10^{-9}$. Because of this, the first order part of $\frac{\mathrm{dlog} F_{\mathrm{e}, \beta}}{\mathrm{d} u}\left(u_{0}\right)$ can safely be neglected.

The off-diagonal terms $o(\beta, \delta)$ can be expanded to the lowest order of $\varepsilon$ as

$o(\beta, \delta) \approx \frac{1}{2}\left(\frac{\varepsilon}{2}\right)^{|\delta|} \exp \left(-i \delta v_{0}\right) \frac{{ }_{0} F_{1}\left(; 1+|\beta+\delta| ;\left(\frac{\pi R}{2 L}\right)^{2}\left(1-\alpha_{\mathrm{i}}^{2}\right)\right)}{{ }_{0} F_{1}\left(; 1+|\beta| ;\left(\frac{\pi R}{2 L}\right)^{2}\left(1-\alpha_{\mathrm{i}}^{2}\right)\right)}\left(\alpha_{\mathrm{i}}^{2}-\alpha_{\mathrm{e}}^{2}\right)$.

In the first order of $\varepsilon$, the only remaining off-diagonal terms are next to the main diagonal. Thus, the resulting system is, in the first order of $\varepsilon$, tri-diagonal. If we want a non-trivial solution of the system, the determinant has to be zero. This gives us a dispersion relation, which eventually leads to the eigenfrequency of the quasi-mode.

\subsection{Modifications to eigenfrequencies}

When dropping higher order terms, system (16) reduces to a homogeneous matrix equation for $D_{\mathrm{i} / \mathrm{e}, \beta}$ with a tri-diagonal structure. Non-trivial solutions only exist when the discriminant of the matrix is zero.

In general the diagonal terms can be written as $d_{0}(\omega)+\varepsilon d_{1}(\omega)$. The only surviving off-diagonal terms can be rewritten as $\varepsilon o_{1}(\omega)$. We assume that $\omega=\omega_{0}+\varepsilon \omega_{1}$. The zeroth order dispersion relation becomes:

$d_{0}\left(\omega_{0}\right)=0$.

This equation can be solved numerically and determines $\omega_{0}$, the eigenfrequency of an uncurved coronal loop. The first order dispersion relation becomes:

$\varepsilon d_{1}\left(\omega_{0}\right)+\varepsilon \omega_{1} \frac{\partial d_{0}}{\partial \omega}\left(\omega_{0}\right)=0$

This gives the expression for $\omega_{1}$ :

$\omega_{1}=-\frac{d_{1}\left(\omega_{0}\right)}{\frac{\partial d_{0}}{\partial \omega}\left(\omega_{0}\right)}$.

However, in our case $d_{1} \equiv 0$. As a consequence, $\omega_{1}=0$.

Hence, in the first order of small curvature, quasi-mode frequencies are unchanged. We also see that the phase $v_{0}$ does not influence the frequency in the first order of the curvature. In higher order, however, quasi-mode frequencies do depend on the oscillation direction (which is determined by $v_{0}$ ).

\subsection{Modifications to eigenmodes}

Although we have no first order corrections to the quasi-mode frequencies, the eigenfunctions do change in the first order. We obtain:

$D_{i, \beta-1}=-\frac{o(\beta,-1)}{d_{0, \beta-1}}$,

$D_{i, \beta+1}=-\frac{o(\beta, 1)}{d_{0, \beta+1}}$.

Here, $d_{0, \beta}$ denotes the zeroth order diagonal term on position $\beta$. Every quantity on the right hand side should be evaluated at $\omega=\omega_{0}$.

We can conclude that, in the first order of $\varepsilon$, quasi-modes only couple to neighbouring poloidal mode numbers. This is consistent with the result found for Alfvén continuum modes by Kieras \& Tataronis (1982).

\section{Solutions including an inhomogeneous layer}

We now insert a layer with a radially varying Alfvén frequency between the two homogeneous regions. In this region, the frequency $\omega$ matches the local Alfvén frequency at some $u$-value. As a consequence, resonant absorption takes place. 
We take a density profile of the form

$$
\rho(u, v)= \begin{cases}\rho_{\mathrm{a}, \mathrm{e}} \frac{(\cosh u-\cos v)^{4}}{\sinh ^{4} u} & \text { for } u<u_{0}, \\ \rho_{\mathrm{a}, \mathrm{i}} \mathrm{b}(u) \frac{(\cosh u-\cos v)^{4}}{\sinh ^{4} u} & \text { for } u_{0} \leq u \leq u_{0}+d, \\ \rho_{\mathrm{a}, \mathrm{i}} \frac{(\cosh u-\cos v)^{4}}{\sinh ^{4} u} & \text { for } u>u_{0}+d .\end{cases}
$$

In order to have a continuous density profile, the conditions $b\left(u_{0}+d\right)=1$ and $b\left(u_{0}\right)=\chi=\frac{\rho_{\mathrm{a}, \mathrm{e}}}{\rho_{\mathrm{a} i}}$ must be fulfilled. The form of this profile is not of great importance. As shown by Van Doorsselaere et al. (2004) the only difference in the complex part of the frequency is multiplication of the damping rate with a constant.

When $d \ll u_{0}$, variation with respect to the spatial coordinate of the perturbed pressure can be neglected in the inhomogeneous region. Consequently, $\left[b_{\phi} / B\right]=0$, where $[Q]=Q_{\mathrm{i}}-Q_{\mathrm{e}}$ denotes the jump of a physical quantity $Q$ across the inhomogeneous layer. This can also be seen by taking the $u$-component of Eq. (4):

$\left(\omega^{2}-\omega_{\mathrm{A}}^{2}\right) V_{u}=V_{\mathrm{A}}^{2} \frac{\cosh u-\cos v}{a} \mathrm{i} \omega \frac{\partial}{\partial u} \frac{b_{\phi}}{B}$

In the neighbourhood of the resonance the left-hand side of the equation is zero. Consequently, $\left[b_{\phi} / B\right]=0$.

Contrary to the pressure, the radial speed does have a jump. To calculate this jump, we eliminate $V_{v}$ from Eq. (5). The resulting equation is:

$\frac{\partial}{\partial u}\left(\frac{V_{u}}{\sinh u}\right)=\mathrm{i} \omega a\left\{\frac{1}{\sinh u(\cosh u-\cos v)} \frac{b_{\phi}}{B}-\frac{\omega_{\mathrm{A}}^{2}}{\omega^{2}-\omega_{\mathrm{A}}^{2}} \frac{\sinh (u)}{k^{2}} \frac{\partial}{\partial v}\left(\frac{1}{\cosh u-\cos v} \frac{\partial}{\partial v} \frac{b_{\phi}}{B}\right)\right\}$.

Neglecting all the non-singular terms gives us the expression for the jump:

$\left[V_{u}\right]=-\frac{\pi a|\omega|}{k^{2}} \frac{\omega_{\mathrm{A}}^{2}}{|\Delta|} \frac{\sinh ^{2} u}{\cosh u-\cos v}\left(\frac{\partial^{2}}{\partial v^{2}} \frac{b_{\phi}}{B}-\frac{\sin v}{\cosh u-\cos v} \frac{\partial}{\partial v} \frac{b_{\phi}}{B}\right)$,

where $\Delta=-\partial \omega_{\mathrm{A}}^{2} / \partial u$. All the quantities ought to be evaluated at the Alfvén resonance position $u_{\mathrm{A}}$, the position where the frequency $\omega$ equals the local Alfvén frequency.

The matching conditions (14) now change to:

$$
\begin{aligned}
& \frac{\mathrm{i} \pi \omega_{\mathrm{A}}^{2}}{|\Delta|}\left(\alpha_{\mathrm{i}}^{2}-1\right)\left(\alpha_{\mathrm{e}}^{2}-1\right) C_{\mathrm{i}, \beta}\left(\beta^{2}+\frac{1}{2} \operatorname{coth} u_{\mathrm{A}}+\frac{3}{4} \frac{1}{\sinh u_{\mathrm{A}}}\right) \\
& \quad+\frac{\mathrm{i} \pi \omega_{\mathrm{A}}^{2}}{|\Delta|}\left(\alpha_{\mathrm{i}}^{2}-1\right)\left(\alpha_{\mathrm{e}}^{2}-1\right) \sum_{\delta \neq 0} C_{\mathrm{i}, \beta+\delta} \frac{F_{\mathrm{i}, \beta+\delta}\left(u_{0}\right)}{F_{\mathrm{i}, \beta}\left(u_{0}\right)} \exp \left(-\mathrm{i} \delta v_{0}\right) \frac{\exp \left(-|\delta| u_{\mathrm{A}}\right)}{2}\left(\operatorname{coth} u_{\mathrm{A}}+\frac{3}{4}(\delta+1) \frac{1}{\sinh u_{\mathrm{A}}}\right) \\
& =C_{\mathrm{i}, \beta}\left(\left(\alpha_{\mathrm{i}}^{2}-1\right) \frac{\mathrm{d} \log F_{\mathrm{e}, \beta}}{\mathrm{d} u}\left(u_{0}\right)-\left(\alpha_{\mathrm{e}}^{2}-1\right) \frac{\mathrm{d} \log F_{\mathrm{i}, \beta}}{\mathrm{d} u}\left(u_{0}\right)+\frac{1-\operatorname{coth} u_{0}}{2}\left(\alpha_{\mathrm{i}}^{2}-\alpha_{\mathrm{e}}^{2}\right)\right) \\
& \quad+\sum_{\delta \neq 0} C_{\mathrm{i}, \beta+\delta} \exp \left(-i \delta v_{0}\right) \frac{\exp \left(-|\delta| u_{0}\right)}{2} \frac{F_{\mathrm{i}, \beta+\delta}\left(u_{0}\right)}{F_{\mathrm{i}, \beta}\left(u_{0}\right)}\left(\alpha_{\mathrm{i}}^{2}-\alpha_{\mathrm{e}}^{2}\right)
\end{aligned}
$$

This is again an infinite system of equations, which can be expanded in $\varepsilon$. Because $d \ll u_{0}, u_{\mathrm{A}} \approx u_{0}$.

We now have two effects, the first due to the resonant layer, the second due to the curvature. If we assume that these effects are both small, the correction to the cylindrical, undamped (real) frequency $\omega_{0 \mathrm{r}}$ is:

$$
\omega_{1}=\frac{\frac{\mathrm{i} \pi \omega_{\mathrm{A}}^{2}}{|\Delta|}\left(\alpha_{\mathrm{i}}^{2}-1\right)\left(\alpha_{\mathrm{e}}^{2}-1\right)\left(\beta^{2}+\frac{1}{2}\right)+\varepsilon \frac{\mathrm{i} \pi \omega_{\mathrm{A}}^{2}}{|\Delta|}\left(\alpha_{\mathrm{i}}^{2}-1\right)\left(\alpha_{\mathrm{e}}^{2}-1\right) \frac{3}{4}}{\left.\left(\frac{\partial}{\partial \omega} d_{0}\right)\right|_{\omega=\omega_{0 \mathrm{r}}}} .
$$

The first term in the numerator is due to resonant absorption, whereas the second term is due to curvature. The resulting $\omega$ can be rewritten as:

$\omega=\omega_{0 \mathrm{r}}-\mathrm{i} \omega_{0 \mathrm{i}}\left(1+\varepsilon \frac{\frac{3}{4}}{\beta^{2}+\frac{1}{2}}\right)$ 
Here $\omega_{0 \mathrm{r}}-\mathrm{i} \omega_{0 \mathrm{i}}$ is the complex frequency of the damped quasi-mode in a cylindrical configuration $(\varepsilon=0)$ and

$\omega_{0 \mathrm{i}}=-\frac{\frac{\pi \omega_{\mathrm{A}}^{2}}{|\Delta|}\left(\alpha_{\mathrm{i}}^{2}-1\right)\left(\alpha_{\mathrm{e}}^{2}-1\right)\left(\beta^{2}+\frac{1}{2}\right)}{\left.\left(\frac{\partial}{\partial \omega} d_{0}\right)\right|_{\omega=\omega_{0 \mathrm{r}}}}$

is the modification due to resonant absorption in the intermediate layer.

For now, we can conclude that quasi-modes are slightly more damped in curved configurations than in cylindrical models.

Caution is required when using these results. In the analysis, we used the fact that $R / L$ is a constant and of the order 1 . However, for realistic coronal loops this is not the case. The previous analytic results may only be applied to low-lying "fat" coronal loops. An analysis for realistic coronal loop parameters is performed in the next section.

\section{Realistic coronal loops}

The previous analysis is not valid for realistic coronal loops. In, as far as curvature is concerned, realistic coronal loops, $k$ does not go to infinity. For the fundamental mode, $k \approx 1$. Therefore, we cannot use the above expansions in small $\varepsilon$. Moreover, when $k \approx 1, R / L$ is of the order of $\varepsilon$. A different analysis has to be used.

Surprisingly, the analysis becomes much simpler in this case. Again we use $\varepsilon$ as a small parameter. However, instead of making $k$ large, we now make $R / L$ small (see Eq. (15)).

In case of an inhomogeneous layer, the diagonal terms become (for $\beta \neq 0$ ):

$-|\beta|\left(2-\alpha_{\mathrm{i}}^{2}-\alpha_{\mathrm{e}}^{2}\right)-\mathrm{i} \frac{\pi \omega_{\mathrm{A}}^{2}}{|\Delta|}\left(\alpha_{\mathrm{i}}^{2}-1\right)\left(\alpha_{\mathrm{e}}^{2}-1\right)\left(\beta^{2}+\frac{1}{2}\right)-\varepsilon \mathrm{i} \frac{\pi \omega_{\mathrm{A}}^{2}}{|\Delta|}\left(\alpha_{\mathrm{i}}^{2}-1\right)\left(\alpha_{\mathrm{e}}^{2}-1\right) \frac{3}{4}$.

To highest order, the off-diagonal terms are in this case:

$\frac{1}{2}\left(\frac{\varepsilon}{2}\right)^{|\delta|} \exp \left(-\mathrm{i} \delta v_{0}\right)\left[\alpha_{\mathrm{i}}^{2}-\alpha_{\mathrm{e}}^{2}-\mathrm{i} \frac{\pi \omega_{\mathrm{A}}^{2}}{|\Delta|}\left(\alpha_{\mathrm{i}}^{2}-1\right)\left(\alpha_{\mathrm{e}}^{2}-1\right)\right]$.

First order corrections to the uncurved frequency do occur. We have $\omega=\omega_{0 \mathrm{r}}-\mathrm{i} \omega_{0 \mathrm{i}}+\varepsilon \delta \omega$, where $\omega_{0 \mathrm{r}}$ is the frequency of the quasi-mode in an uncurved configuration without resonant absorption. Similarly to the previous section, if we assume that the two modifications to $\omega_{0 \mathrm{r}}$ are small, we can write for realistic coronal loops

$i \omega_{0 \mathrm{i}}+\varepsilon \delta \omega=\frac{i \frac{\pi \omega_{0 \mathrm{r}}^{2}}{|\Delta|}\left(\frac{\omega_{0 \mathrm{r}}^{2}}{\omega_{\mathrm{Ai}}^{2}}-1\right)\left(\frac{\omega_{0 \mathrm{r}}^{2}}{\omega_{\mathrm{Ae}}^{2}}-1\right)\left(\beta^{2}+\frac{1}{2}\right)+\varepsilon i \frac{\pi \omega_{0 \mathrm{r}}^{2}}{|\Delta|}\left(\frac{\omega_{0 \mathrm{r}}^{2}}{\omega_{\mathrm{Ai}}^{2}}-1\right)\left(\frac{\omega_{0 \mathrm{r}}^{2}}{\omega_{\mathrm{Ae}}^{2}}-1\right) \frac{3}{4}}{2|\beta| \omega_{0 \mathrm{r}}\left(\frac{\omega_{\mathrm{Ae}}^{2}+\omega_{\mathrm{Ai}}^{2}}{\omega_{\mathrm{Ae}}^{2} \omega_{\mathrm{Ai}}^{2}}\right)}$.

Analogous to the previous section, the complex frequency of the damped quasi-mode in realistic coronal loop configurations can be written as

$\omega=\omega_{0 \mathrm{r}}-\mathrm{i} \omega_{0 \mathrm{i}}\left(1+\varepsilon \frac{\frac{3}{4}}{\beta^{2}+\frac{1}{2}}\right)$

where

$\omega_{0 \mathrm{r}}=\frac{2 \omega_{\mathrm{Ae}}^{2} \omega_{\mathrm{Ai}}^{2}}{\omega_{\mathrm{Ae}}^{2}+\omega_{\mathrm{Ai}}^{2}}$,
$\omega_{0 \mathrm{i}}=-\frac{\frac{\pi \omega_{0 \mathrm{r}}^{2}}{|\Delta|}\left(\frac{\omega_{0 \mathrm{r}}^{2}}{\omega_{\mathrm{Ai}}^{2}}-1\right)\left(\frac{\omega_{0 \mathrm{r}}^{2}}{\omega_{\mathrm{Ae}}^{2}}-1\right)\left(\beta^{2}+\frac{1}{2}\right)}{2|\beta| \omega_{0 \mathrm{r}}\left(\frac{\omega_{\mathrm{Ae}}^{2}+\omega_{\mathrm{Ai}}^{2}}{\omega_{\mathrm{Ae}}^{2} \omega_{\mathrm{Ai}}^{2}}\right)}$.

As a result, we can conclude that quasi-modes are more damped in curved configurations.

Substituting observed coronal loop parameters (Aschwanden et al. 2002) in Eq. (21), the increase of the imaginary part of the frequency is at maximum $12 \%$ and generally less than $5 \%$ for kink modes $(\beta= \pm 1)$. For higher poloidal wave numbers $\beta$ the increase is even less. 


\section{Conclusions}

From this paper we can conclude that ideal quasi-modes in coronal loops are not much influenced by longitudinal curvature. The oscillation frequency remains unaffected by the curvature. On the contrary, quasi-modes in coronal loops are slightly more damped in curved configurations. Furthermore, the eigenmodes couple in the first order of the curvature only to neighbouring poloidal mode numbers. This coupling is analogous with the coupling of poloidal Alfvén continuum modes in tokamak devices (Kieras \& Tataronis 1982).

In this paper, we only use first order correction due to curvature. Second order terms can be neglected because, for the coronal loops observed by Aschwanden et al. (2002), $\varepsilon$ is at most 0.24 and generally smaller than 0.1 . Moreover, since these values for $\varepsilon$ are so small for most of the observed oscillating coronal loops, it is often safe to use a cylindrical model instead of a curved one. For kink modes the deviation from the cylindrical frequency is smaller than $12 \%$.

Another important conclusion is that curvature does not select a preferential oscillation direction. For the solution in the poloidal direction, any phase is allowed. Thus, the oscillation may differ depending on the impact direction of the triggering event. This implies that, for certain phases, quasi-modes should also be visible in the Doppler-shifts, when looking at the top of a coronal loop. Such oscillations were first observed by Wang \& Solanki (2004).

\section{References}

Abramowitz, M., \& Stegun, I. A. 1964, Handbook of Mathematical Functions (Washington DC: National Bureau of Standards)

Aschwanden, M. J., De Pontieu, B., Schrijver, C. J., \& Title, A. M. 2002, Sol. Phys., 206, 99

Aschwanden, M. J., Fletcher, L., Schrijver, C. J., \& Alexander, D. 1999, ApJ, 520, 880

Aschwanden, M. J., Nightingale, R. W., Andries, J., Goossens, M., \& Van Doorsselaere, T. 2003, ApJ, 598, 1375

Goedbloed, J. P., \& Halberstadt, G. 1994, A\&A, 286, 275

Goossens, M., Andries, J., \& Aschwanden, M. J. 2002a, A\&A, 394, L39

Goossens, M., De Groof, A., \& Andries, J. 2002b, in SP-505 Magnetic coupling of the solar atmosphere,Proc. of IAU Coll., ed. H. SawayaLacoste (Noordwijk, The Netherlands: ESA), 188, 137

Goossens, M., Hollweg, J. V., \& Sakurai, T. 1992, Sol. Phys., 138, 233

Goossens, M., Ruderman, M. S., \& Hollweg, J. V. 1995, Sol. Phys., 157, 75

Hollweg, J. V. 1990, J. Geophys. Res., 95, 2319

Hollweg, J. V., \& Yang, G. 1988, J. Geophys. Res., 93, 5423

Kieras, C., \& Tataronis, J. 1982, J. Plasma Phys., 28, 395

Nakariakov, V. M., \& Ofman, L. 2001, A\&A, 372, L53

Nakariakov, V. M., Ofman, L., DeLuca, E. E., Roberts, B., \& Davila, J. M. 1999, Science, 285, 862

Ruderman, M. S., \& Roberts, B. 2002, ApJ, 577, 475

Schrijver, C. J., Aschwanden, M. J., \& Title, A. M. 2002, Sol. Phys., 206, 69

Schrijver, C. J., Title, A. M., Berger, T. E., et al. 1999, Sol. Phys., 187, 261

Van Doorsselaere, T., Andries, J., Poedts, S., \& Goossens, M. 2004, ApJ, 606, 1223

Wang, T. J., \& Solanki, S. K. 2004, A\&A, 421, L33 
T. Van Doorsselaere et al.: The effect of curvature on quasi-modes, Online Material p 1

\section{Online Material}




\section{Appendix A: Expansions for $\frac{\mathrm{dlog} F_{\mathrm{e}, \beta^{\prime}}}{\mathrm{d} u}$}

In Eq. (16) an expansion of the solution for small curvature $(\varepsilon \ll 1)$ was performed. However, we only showed the expansions for the internal solutions. For completeness, we list in this appendix the expansions (for small $\varepsilon$ ) of the external solution, i.e. we expand the term in Eq. (16) containing $\frac{\mathrm{dlog} F_{\mathrm{e}, \beta^{\prime}}}{\mathrm{d} u}$.

$$
\begin{aligned}
\frac{\mathrm{d} \log F_{\mathrm{e}, \beta^{\prime}}}{\mathrm{d} u}= & \frac{\mathrm{d}}{\mathrm{d} u}\left\{\left(-\frac{1}{2}-k \sqrt{1-\alpha_{\mathrm{e}}^{2}}\right) \log \operatorname{coth} u-\beta \log \cosh u\right. \\
& \left.+\log _{2} F_{1}\left(\frac{1}{4}+\frac{k}{2} \sqrt{1-\alpha_{\mathrm{e}}^{2}}+\frac{\beta}{2}, \frac{3}{4}+\frac{k}{2} \sqrt{1-\alpha_{\mathrm{e}}^{2}}+\frac{\beta}{2} ; 1+k \sqrt{1-\alpha_{\mathrm{e}}^{2}} ; \tanh ^{2} u\right)\right\} \\
= & -\beta+\operatorname{term}_{2} F_{1}+\varepsilon\left(\frac{\pi R}{L} \sqrt{1-\alpha_{\mathrm{e}}^{2}}+\operatorname{term}_{2} F_{1}\right) .
\end{aligned}
$$

To determine the contributions from the term with the hypergeometric functions $\left(\right.$ term $\left.F_{1}\right)$, we have to use the analytic continuation of the function around 1. Because the third parameter then becomes an integer plus the sum of the two other parameters, we need to distinguish between cases (Abramowitz \& Stegun 1964). However, for all the cases, the expansion takes the same form. In general, we can write:

$$
\frac{\mathrm{d}}{\mathrm{d} u} \log _{2} F_{1}\left(\frac{1}{4}+\frac{k}{2} \sqrt{1-\alpha_{\mathrm{e}}^{2}}+\frac{\beta}{2}, \frac{3}{4}+\frac{k}{2} \sqrt{1-\alpha_{\mathrm{e}}^{2}}+\frac{\beta}{2} ; 1+k \sqrt{1-\alpha_{\mathrm{e}}^{2}} ; \tanh ^{2} u\right) \approx\left(V_{\beta}^{0}+\varepsilon V_{\beta}^{1}\right) \frac{E_{\beta}^{0}+\varepsilon E_{\beta}^{1}}{D_{\beta}^{0}+\varepsilon D_{\beta}^{1}}
$$

Rearranging the terms, we get in first order:

$$
\begin{aligned}
\frac{\mathrm{d}}{\mathrm{d} u} \log _{2} F_{1} & =\left(V_{\beta}^{0}+\varepsilon V_{\beta}^{1}\right) \frac{E_{\beta}^{0}+\varepsilon E_{\beta}^{1}}{D_{\beta}^{0}+\varepsilon D_{\beta}^{1}} \\
& =V_{\beta}^{0} \frac{E_{\beta}^{0}}{D_{\beta}^{0}}\left(1+\varepsilon\left(\frac{V_{\beta}^{1}}{V_{\beta}^{0}}+\frac{E_{\beta}^{1}}{E_{\beta}^{0}}-\frac{D_{\beta}^{1}}{D_{\beta}^{0}}\right)\right) .
\end{aligned}
$$

When $\beta>0$, we have:

$$
\begin{aligned}
V_{\beta}^{0}= & 2 \beta, \\
V_{\beta}^{1}= & 0 \\
E_{\beta}^{0}= & \sum_{n=0}^{\beta} \frac{1}{n !(-\beta)_{n}}\left(\left(\frac{\pi R}{2 L}\right)^{2}\left(1-\alpha_{\mathrm{e}}^{2}\right)\right)^{n}+\frac{(-1)^{\beta}}{\Gamma(\beta+1)}\left(\left(\frac{\pi R}{2 L}\right)^{2}\left(1-\alpha_{\mathrm{e}}^{2}\right)\right)^{(\beta+1)} \\
& \times \sum_{n=0}^{\infty} \frac{1}{n !(n+\beta+1) !}\left(\left(\frac{\pi R}{2 L}\right)^{2}\left(1-\alpha_{\mathrm{e}}^{2}\right)\right)^{n}\left[\log \left(\left(\frac{\pi R}{2 L}\right)^{2}\left(1-\alpha_{\mathrm{e}}^{2}\right)\right)-\Psi(n+1)-\Psi(n+\beta+2)\right], \\
E_{\beta}^{1}= & \sum_{n=0}^{\beta} \frac{n(n-\beta)}{n !(-\beta)_{n}}\left(\frac{\pi R}{2 L} \sqrt{1-\alpha_{\mathrm{e}}^{2}}\right)^{2 n-1}+\frac{(-1)^{\beta}}{\Gamma(\beta+1)}\left(\frac{\pi R}{2 L} \sqrt{1-\alpha_{\mathrm{e}}^{2}}\right)^{(2 \beta+1)} \\
& \times \sum_{n=0}^{\infty} \frac{1}{n !(n+\beta+1) !}\left(\left(\frac{\pi R}{2 L}\right)^{2}\left(1-\alpha_{\mathrm{e}}^{2}\right)\right)^{n}(2 n+\beta+2+n(n+\beta+2) \\
& \left.\times\left[\log \left(\left(\frac{\pi R}{2 L}\right)^{2}\left(1-\alpha_{\mathrm{e}}^{2}\right)\right)-\Psi(n+1)-\Psi(n+\beta+2)\right]\right)+\frac{(-1)^{\beta}(\beta+1)}{\Gamma(\beta+1)}\left(\frac{\pi R}{2 L} \sqrt{1-\alpha_{\mathrm{e}}^{2}}\right) \\
& \times \sum_{n=0}^{\infty} \frac{1}{n !(n+\beta+1)} \\
& \beta-1 \\
D_{\beta}^{0}= & \sum_{n=0} \frac{1}{n !(1-\beta)_{n}}\left(\left(\frac{\pi R}{2 L}\right)^{2}\left(1-\alpha_{\mathrm{e}}^{2}\right)\right)^{n}+\frac{(-1)^{\beta-1}}{\Gamma(\beta)}\left(\left(\frac{\pi R}{2 L}\right)^{2}\left(1-\alpha_{\mathrm{e}}^{2}\right)\right)^{\beta} \frac{1}{n !(n+\beta) !}\left(\left(\frac{\pi R}{2 L}\right)^{2}\left(1-\alpha_{\mathrm{e}}^{2}\right)\right)^{n}\left[\log \left(\left(\frac{\pi R}{2 L}\right)^{2}\left(1-\alpha_{\mathrm{e}}^{2}\right)\right)-\Psi(n+1)-\Psi(n+\beta+1)\right], \\
& \left.\times \sum^{2}\left(1-\alpha_{\mathrm{e}}^{2}\right)\right)^{n}\left[\log \left(\left(\frac{\pi R}{2 L}\right)^{2}\left(1-\alpha_{\mathrm{e}}^{2}\right)\right)-\Psi(n+1)-\Psi(n+\beta+2)\right],
\end{aligned}
$$


T. Van Doorsselaere et al.: The effect of curvature on quasi-modes, Online Material p 3

$$
\begin{aligned}
D_{\beta}^{1}= & \sum_{n=0}^{\beta-1} \frac{n(n-\beta)}{n !(1-\beta)_{n}}\left(\frac{\pi R}{2 L} \sqrt{1-\alpha_{\mathrm{e}}^{2}}\right)^{2 n-1}+\frac{(-1)^{\beta-1}}{\Gamma(\beta)}\left(\frac{\pi R}{2 L} \sqrt{1-\alpha_{\mathrm{e}}^{2}}\right)^{(2 \beta-1)} \\
& \times \sum_{n=0}^{\infty} \frac{1}{n !(n+\beta) !}\left(\left(\frac{\pi R}{2 L}\right)^{2}\left(1-\alpha_{\mathrm{e}}^{2}\right)\right)^{n}\left(2 n+\beta+n(n+\beta)\left[\log \left(\left(\frac{\pi R}{2 L}\right)^{2}\left(1-\alpha_{\mathrm{e}}^{2}\right)\right)-\Psi(n+1)-\Psi(n+\beta+1)\right]\right) .
\end{aligned}
$$

If $\beta<-1$, we have the following formulae:

$$
\begin{aligned}
V_{\beta}^{0}= & -\frac{2}{\beta+1}\left(\left(\frac{\pi R}{2 L}\right)^{2}\left(1-\alpha_{\mathrm{e}}^{2}\right)\right), \\
V_{\beta}^{1}= & -\frac{\pi R}{L} \sqrt{1-\alpha_{\mathrm{e}}^{2}}, \\
E_{\beta}^{0}= & \sum_{n=0}^{-\beta-2} \frac{1}{n !(2+\beta)_{n}}\left(\left(\frac{\pi R}{2 L}\right)^{2}\left(1-\alpha_{\mathrm{e}}^{2}\right)\right)^{n}+\frac{(-1)^{-\beta}}{\Gamma(-\beta-1)}\left(\left(\frac{\pi R}{2 L}\right)^{2}\left(1-\alpha_{\mathrm{e}}^{2}\right)\right)^{(-\beta-1)} \\
& \times \sum_{n=0}^{\infty} \frac{1}{n !(n-\beta-1) !}\left(\left(\frac{\pi R}{2 L}\right)^{2}\left(1-\alpha_{\mathrm{e}}^{2}\right)\right)^{n}\left[\log \left(\left(\frac{\pi R}{2 L}\right)^{2}\left(1-\alpha_{\mathrm{e}}^{2}\right)\right)-\Psi(n+1)-\Psi(n-\beta)\right], \\
E_{\beta}^{1}= & \sum_{n=0} \frac{n-2}{n !(\beta+n+2)}\left(\frac{\pi R}{2 L} \sqrt{1-\alpha_{\mathrm{e}}^{2}}\right)^{2 n-1}+\frac{(-1)^{-\beta}}{\Gamma(-\beta-1)}\left(\frac{\pi R}{2 L} \sqrt{1-\alpha_{\mathrm{e}}^{2}}\right)^{(2 \beta-3)} \\
& \times \sum_{n=0}^{\infty} \frac{1}{n !(n-\beta-1) !}\left(\left(\frac{\pi R}{2 L}\right)^{2}\left(1-\alpha_{\mathrm{e}}^{2}\right)\right)^{n}(2 n-\beta+n(n-\beta) \\
& \left.\times\left[\log \left(\left(\frac{\pi R}{2 L}\right)^{2}\left(1-\alpha_{\mathrm{e}}^{2}\right)\right)^{-}-\Psi(n+1)-\Psi(n-\beta)\right]\right)+\frac{(-1)^{-\beta}(-\beta-1)}{\Gamma(-\beta-1)}\left(\frac{\pi R}{2 L} \sqrt{1-\alpha_{\mathrm{e}}^{2}}\right) \\
& \times \sum_{n=0}^{\infty} \frac{1}{n !(n-\beta-1) !}\left(\left(\frac{\pi R}{2 L}\right)^{2}\left(1-\alpha_{\mathrm{e}}^{2}\right)\right)^{n}\left[\log \left(\left(\frac{\pi R}{2 L}\right)^{2}\left(1-\alpha_{\mathrm{e}}^{2}\right)\right)-\Psi(n+1)-\Psi(n-\beta)\right], \\
D_{\beta}^{0}= & \sum_{n=0}^{-\beta-1} \frac{1}{n !(1+\beta)_{n}}\left(\left(\frac{\pi R}{2 L}\right)^{2}\left(1-\alpha_{\mathrm{e}}^{2}\right)\right)^{n}+\frac{(-1)^{-\beta-1}}{\Gamma(-\beta)}\left(\left(\frac{\pi R}{2 L}\right)^{2}\left(1-\alpha_{\mathrm{e}}^{2}\right)\right)^{-\beta} \\
& \times \sum_{n=0}^{\infty} \frac{1}{n !(n-\beta) !}\left(\left(\frac{\pi R}{2 L}\right)^{2}\left(1-\alpha_{\mathrm{e}}^{2}\right)\right)^{n}\left[\log \left(\left(\frac{\pi R}{2 L}\right)^{2}\left(1-\alpha_{\mathrm{e}}^{2}\right)\right)-\Psi(n+1)-\Psi(n-\beta+1)\right], \\
& -\beta-1 \\
D_{\beta}^{1}= & \sum_{n=0} \frac{n(\beta+n)}{n !(1+\beta)_{n}}\left(\frac{\pi R}{2 L} \sqrt{1-\alpha_{\mathrm{e}}^{2}}\right)^{2 n-1}+\frac{(-1)^{-\beta-1}}{\Gamma(-\beta)}\left(\frac{\pi R}{2 L} \sqrt{1-\alpha_{\mathrm{e}}^{2}}\right)^{(-2 \beta-1)} \\
& \times \sum_{n=0}^{\infty} \frac{1}{n !(n-\beta) !}\left(\left(\frac{\pi R}{2 L}\right)^{2}\left(1-\alpha_{\mathrm{e}}^{2}\right)\right)^{n}\left(2 n-\beta+n(n-\beta)\left[\log \left(\left(\frac{\pi R}{2 L}\right)^{2}\left(1-\alpha_{\mathrm{e}}^{2}\right)\right)-\Psi(n+1)-\Psi(n-\beta+1)\right]\right)
\end{aligned}
$$

For $\beta=-1$, we have:

$$
\begin{aligned}
V_{\beta}^{0}= & 2\left(\frac{\pi R}{2 L}\right)^{2}\left(1-\alpha_{\mathrm{e}}^{2}\right), \\
V_{\beta}^{1}= & 0, \\
E_{\beta}^{0}= & -\sum_{n=0}^{\infty} \frac{1}{(n !)^{2}}\left(\left(\frac{\pi R}{2 L}\right)^{2}\left(1-\alpha_{\mathrm{e}}^{2}\right)\right)^{n}\left[\log \left(\left(\frac{\pi R}{2 L}\right)^{2}\left(1-\alpha_{\mathrm{e}}^{2}\right)\right)-2 \Psi(n+1)\right], \\
E_{\beta}^{1}= & -\frac{1}{\frac{\pi R}{2 L} \sqrt{1-\alpha_{\mathrm{e}}^{2}}} \sum_{n=0}^{\infty} \frac{1}{(n !)^{2}}\left(\left(\frac{\pi R}{2 L}\right)^{2}\left(1-\alpha_{\mathrm{e}}^{2}\right)\right)^{n}\left(2 n+1+n(n+1)\left[\log \left(\left(\frac{\pi R}{2 L}\right)^{2}\left(1-\alpha_{\mathrm{e}}^{2}\right)\right)-2 \Psi(n+1)\right]\right), \\
D_{\beta}^{0}= & 1+\left(\frac{\pi R}{2 L}\right)^{2}\left(1-\alpha_{\mathrm{e}}^{2}\right) \sum_{n=0}^{\infty} \frac{1}{n !(n+1) !}\left(\left(\frac{\pi R}{2 L}\right)^{2}\left(1-\alpha_{\mathrm{e}}^{2}\right)\right)^{n}\left[\log \left(\left(\frac{\pi R}{2 L}\right)^{2}\left(1-\alpha_{\mathrm{e}}^{2}\right)\right)-\Psi(n+1)-\Psi(n+2)\right], \\
D_{\beta}^{1}= & \frac{\pi R}{2 L} \sqrt{1-\alpha_{\mathrm{e}}^{2}} \sum_{n=0}^{\infty} \frac{1}{n !(n+1) !}\left(\left(\frac{\pi R}{2 L}\right)^{2}\left(1-\alpha_{\mathrm{e}}^{2}\right)\right)^{n}(2 n+1+n(n+1) \\
& \left.\times\left[\log \left(\left(\frac{\pi R}{2 L}\right)^{2}\left(1-\alpha_{\mathrm{e}}^{2}\right)\right)-\Psi(n+1)-\Psi(n+2)\right]\right) .
\end{aligned}
$$


The last case, $\beta=0$ gives:

$V_{\beta}^{0}=2$,

$V_{\beta}^{1}=0$,

$$
\begin{aligned}
E_{\beta}^{0}= & +\left(\frac{\pi R}{2 L}\right)^{2}\left(1-\alpha_{\mathrm{e}}^{2}\right) \sum_{n=0}^{\infty} \frac{1}{n !(n+1) !}\left(\left(\frac{\pi R}{2 L}\right)^{2}\left(1-\alpha_{\mathrm{e}}^{2}\right)\right)^{n}\left[\log \left(\left(\frac{\pi R}{2 L}\right)^{2}\left(1-\alpha_{\mathrm{e}}^{2}\right)\right)-\Psi(n+1)-\Psi(n+2)\right], \\
E_{\beta}^{1}= & \frac{\pi R}{2 L} \sqrt{1-\alpha_{\mathrm{e}}^{2}} \sum_{n=0}^{\infty} \frac{1}{n !(n+1) !}\left(\left(\frac{\pi R}{2 L}\right)^{2}\left(1-\alpha_{\mathrm{e}}^{2}\right)\right)^{n}(2 n+2+n(n+2) \\
& \left.\times\left[\log \left(\left(\frac{\pi R}{2 L}\right)^{2}\left(1-\alpha_{\mathrm{e}}^{2}\right)\right)-\Psi(n+1)-\Psi(n+2)\right]\right)+\frac{\pi R}{2 L} \sqrt{1-\alpha_{\mathrm{e}}^{2}} \\
& \times \sum_{n=0}^{\infty} \frac{1}{n !(n+1) !}\left(\left(\frac{\pi R}{2 L}\right)^{2}\left(1-\alpha_{\mathrm{e}}^{2}\right)\right)^{n}\left[\log \left(\left(\frac{\pi R}{2 L}\right)^{2}\left(1-\alpha_{\mathrm{e}}^{2}\right)\right)-\Psi(n+1)-\Psi(n+2)\right], \\
D_{\beta}^{0}= & -\sum_{n=0}^{\infty} \frac{1}{(n !)^{2}}\left(\left(\frac{\pi R}{2 L}\right)^{2}\left(1-\alpha_{\mathrm{e}}^{2}\right)\right)^{n}\left[\log \left(\left(\frac{\pi R}{2 L}\right)^{2}\left(1-\alpha_{\mathrm{e}}^{2}\right)\right)-2 \Psi(n+1)\right], \\
D_{\beta}^{1}= & -\frac{1}{\frac{\pi R}{2 L} \sqrt{1-\alpha_{\mathrm{e}}^{2}}} \sum_{n=0}^{\infty} \frac{1}{(n !)^{2}}\left(\left(\frac{\pi R}{2 L}\right)^{2}\left(1-\alpha_{\mathrm{e}}^{2}\right)\right)^{n}\left(2 n+n^{2}\left[\log \left(\left(\frac{\pi R}{2 L}\right)^{2}\left(1-\alpha_{\mathrm{e}}^{2}\right)\right)-2 \Psi(n+1)\right]\right) .
\end{aligned}
$$

